\title{
Gliotoxin effects on fungal growth: Mechanisms and exploitation
}

\author{
Stephen Carberry, Emer Molloy, Stephen Hammel, Grainne O’Keeffe, Gary W. Jones, \\ Kevin Kavanagh, Sean Doyle*
}

Department of Biology, National University of Ireland Maynooth, Co. Kildare, Ireland

\section{A R T I C L E I N F O}

\section{Article history:}

Received 12 October 2011

Accepted 10 February 2012

Available online 1 March 2012

\section{Keywords:}

Aspergillus fumigatus

Proteomics

Natural products

Aspergillus

Yeast

NRPS

\begin{abstract}
A B S T R A C T
Although initially investigated for its antifungal properties, little is actually known about the effect of gliotoxin on Aspergillus fumigatus and other fungi. We have observed that exposure of A. fumigatus to exogenous gliotoxin $(14 \mu \mathrm{g} / \mathrm{ml})$, under gliotoxin-limited growth conditions, results in significant alteration of the expression of 27 proteins (up- and down-regulated $>1.9$-fold; $p<0.05$ ) including de novo expression of $\mathrm{Cu}, \mathrm{Zn}$ superoxide dismutase, up-regulated allergen Asp $\mathrm{f} 3$ expression and down-regulated catalase and a peroxiredoxin levels. Significantly elevated glutathione GSH levels $(p<0.05)$, along with concomitant resistance to diamide, were evident in A. fumigatus $\Delta$ gliT, lacking gliotoxin oxidoreductase, a gliotoxin self-protection gene. Saccharomyces cerevisiae deletents ( $\Delta$ sod 1 and $\Delta y a p 1)$ were hypersensitive to exogenous gliotoxin, while $\Delta$ gsh 1 was resistant. Significant gliotoxin-mediated $(5 \mu \mathrm{g} / \mathrm{ml})$ growth inhibition $(p<0.001)$ of Aspergillus nidulans, Aspergillus terreus, Aspergillus niger, Cochliobolus heterostrophus and Neurospora crassa was also observed. Growth of Aspergillus flavus, Fusarium graminearum and Aspergillus oryzae was significantly inhibited $(p<0.001)$ at gliotoxin $(10 \mu \mathrm{g} / \mathrm{ml})$, indicating differential gliotoxin sensitivity amongst fungi. Re-introduction of gliT into A. fumigatus $\Delta$ gliT, at a different locus (ctsD; AFUA_4G07040, an aspartic protease), with selection on gliotoxin, facilitated deletion of $c t s D$ without use of additional antibiotic selection markers. Absence of ctsD expression was accompanied by restoration of gliT expression, and resistance to gliotoxin. Thus, we propose gliT/gliotoxin as a useful selection marker system for fungal transformation. Finally, we suggest incorporation of gliotoxin sensitivity assays into all future fungal functional genomic studies.
\end{abstract}

(c) 2012 Elsevier Inc. All rights reserved.

\section{Introduction}

Production of gliotoxin, an epipolythiodioxopiperazine, by Aspergillus fumigatus is enabled by a $28 \mathrm{~kb}$ gene cluster ( $g l i$ located on chromosome 6 (Gardiner et al., 2004; Cramer et al., 2006). The molecule has been intensively studied because of its cytotoxic, immuno-inhibitory and apoptotic effects on animal cells (Sutton et al., 1994). Gliotoxin has recently been shown to inhibit angiogenesis and it has been proposed that this effect prevents tissue neovascularisation, impedes the proinflammatory response and contributes to tissue damage (Ben-ami et al., 2009). As a result of disulphide bridge presence, gliotoxin is a redox active molecule and can cycle between the oxidised and reduced forms depending on the cellular redox state (Waring et al., 1995). In an elegant set of experiments, Bernardo et al. (2003) demonstrated that the oxidised form of gliotoxin is taken up by mammalian cells, undergoes glutathione (GSH)-mediated reduction to the reduced form, which ultimately re-oxidises upon depletion of intracellular GSH and is

\footnotetext{
* Corresponding author. Fax: +353 17083845.

E-mail address: sean.doyle@nuim.ie (S. Doyle).

URL: http://biology.nuim.ie (S. Doyle).
}

effluxed from the cell. Deletion of gliz (Bok et al., 2006) and gliP (Cramer et al., 2006; Kupfahl et al., 2006), gliT (Schrettl et al., 2010; Scharf et al., 2010) and gliG (Davis et al., 2011) respectively, abolishes gliotoxin production by A. fumigatus. Indeed, Cramer et al. (2006) demonstrated that exogenous gliotoxin controlled the expression of the gli cluster in A. fumigatus and thereby regulated its own production.

Chamilos et al. (2008) studied the effect of gliotoxin on Saccharomyces cerevisiae, using a library of single-gene mutants (4787 strains), in an attempt to further elucidate mechanisms of gliotoxin cytotoxicity and identify novel drug targets in eukaryotic cells. Overall, 10 mutants exhibited increased resistance to gliotoxin while 3 were statistically more sensitive to exogenous gliotoxin, compared to wild-type. Increased resistance to gliotoxin was observed in strains lacking genes encoding metabolic, vesicular transport, DNA damage repair and unknown function functions, while increased sensitivity was evident when Cys3, a cystathionine gamma-lyase and Mef1 a transcriptional elongation factor of mitochondrial origin were absent.

Rohlfs et al. (2007) demonstrated that toxic secondary metabolites produced by Aspergillus nidulans, under control of the global regulator laeA, significantly discouraged feeding by the fungivore, 
Folsomia candida and proposed that fungivory may select for secondary metabolite biosynthesis in fungi. This led Kwon-Chung and Sugui (2009) to note that production of secondary metabolites might improve the survival chances of fungi. Moreover, although secondary metabolite production may also be influenced by competition between different fungal species, until recently, it was not known if secondary metabolites produced by Aspergilli conferred a growth competitive advantage in the presence of related species (Losada et al., 2009). Although these authors identified a number of secreted metabolites, which may contribute to improved survival, no information with respect to individual metabolite potency, or the effect on non-Aspergillus spp. was presented. Losada et al. postulated that resistance genes would be required to allow a toxin producer to grow in the presence of its metabolite weaponry and it has now been shown that gliT in the gli cluster mediates self-protection against exogenous gliotoxin in A. fumigatus (Schrettl et al., 2010; Scharf et al., 2010) whereby a gliT-deficient strain was unable to grow in the presence of gliotoxin. In fact, gliT is the key resistance gene against gliotoxin in A. fumigatus and Schrettl et al. demonstrated that $\Delta g l i T$ complementation was possible using selection on gliotoxin-containing media without additional antibiotics (e.g., hygromycin or phleomycin). However, use of gliT re-introduction into A. fumigatus $\Delta$ gliT, and subsequent selection in gliotoxin presence, for the targeted deletion of unrelated genes has not been demonstrated.

Thus, a new paradigm has emerged where the effects, and role, of gliotoxin in A. fumigatus, in particular, and fungi generally, requires investigation: as it may represent a novel probe to further our understanding of the function of ETPs, and redox control mechanisms, in fungi. In addition, exploitation of the effects of gliotoxin to develop a new fungal selection marker system may be feasible.

\section{Materials and methods}

\subsection{Extraction of gliotoxin from culture filtrates}

A. fumigatus ATCC26933 $\left(1 \times 10^{5} \mathrm{cfu} / \mathrm{ml}\right)$ was grown for $24 \mathrm{~h}$ in either Minimal Essential Medium (plus 5\% (v/v) fetal calf serum $(\mathrm{MEM} / \mathrm{FCS})$ ) or Sabouraud media $\left(25 \mathrm{ml}\right.$ cultures), at $37^{\circ} \mathrm{C}$ with shaking at $200 \mathrm{rpm}$. Supernatants were separated by filtration and an equal volume of extraction buffer (ethyl acetate:chloroform:methanol, 3:2:1) was added. After overnight incubation at $4{ }^{\circ} \mathrm{C}$, filtrates were centrifuged and organic layers removed and evaporated to dryness. Dried extracts were reconstituted in $200 \mu \mathrm{l}$ HPLC grade Methanol and stored at $-70^{\circ} \mathrm{C}$ until assay.

\subsection{RP-HPLC analysis}

Gliotoxin was detected using a reversed phase HPLC (SpectraPhysics). Gradient elution was performed with Solvent A consisting of $0.1 \%(\mathrm{v} / \mathrm{v})$ trifluoroacetic acid in 5:95 acetonitrile:HPLC grade water (Sigma-Aldrich) and Solvent B consisting of $0.1 \%(\mathrm{v} / \mathrm{v})$ trifluoroacetic acid in $99.9 \%$ acetonitrile. Gliotoxin extracts $(20 \mu \mathrm{l})$ were injected onto the $\mathrm{C}_{18}$ column (Hewlett Packard). All cultures were grown up in triplicate and each one analysed in duplicate. Average values were compared to a standard curve (0-1.0 $\mu$ g gliotoxin) and gliotoxin quantified as micrograms of gliotoxin per milligram of mycelia produced \pm standard deviation.

\subsection{Protein extraction}

A. fumigatus ATCC26933 $\left(1 \times 10^{5} \mathrm{cfu} / \mathrm{ml}\right)$ was grown for $24 \mathrm{~h}$ in Sabouraud media (50 ml cultures), at $37^{\circ} \mathrm{C}$ with shaking at $200 \mathrm{rpm}$. After $24 \mathrm{~h}$, gliotoxin $(700 \mu \mathrm{g})$ dissolved in methanol was added to the cultures $(n=3)$. As a control, methanol only was added to parallel cultures $(n=3)$. After $4 \mathrm{~h}$ incubation, mycelia were harvested, filtered under pressure, washed with PBS and resuspended in lysis buffer $(100 \mathrm{mM}$ Tris- $\mathrm{HCl}, 50 \mathrm{mM} \mathrm{NaCl}$, $20 \mathrm{mM}$ EDTA, 10\% (v/v) glycerol, $30 \mathrm{mM}$ DTT, $1 \mathrm{mM}$ PMSF and $1 \mu \mathrm{g} / \mathrm{ml}$ pepstatin A pH 7.5; $3 \mathrm{ml}$ of lysis buffer per gram of mycelia). Lysis was accomplished by grinding in liquid $\mathrm{N}_{2}$ followed by brief sonication on ice. Mycelial lysates were centrifuged $(10,000 \mathrm{~g} ; 30 \mathrm{~min})$ to remove cell debris and the subsequent supernatants analysed by 2D-PAGE following TCA/acetone precipitation (Carberry et al., 2006).

\subsection{Protein quantification}

Protein was quantified using Bradford reagent (BioRad Laboratories) with BSA used as a standard protein.

\subsection{Two-dimensional electrophoresis}

Extracts containing $250 \mu \mathrm{g}$ protein were resuspended in $8 \mathrm{M}$ Urea, $2 \mathrm{M}$ Thiourea, 4\% (w/v) CHAPS, 1\% (v/v) Triton-X-100, $10 \mathrm{mM}$ Tris-HC1, $65 \mathrm{mM}$ DTT, $0.8 \% \mathrm{pH}$ 4-7 carrier ampholytes and loaded onto Immobiline Dry strips (GE Healthcare) in the $\mathrm{pH}$ range 4-7. Following IEF on an IPGphor II, gels were equilibrated in reducing buffer ( $50 \mathrm{mM}$ Tris- $\mathrm{HCl}, 6 \mathrm{M}$ Urea, $2 \%$ (w/v) SDS, 30\% (v/v) Glycerol, 2\% (w/v) DTT, pH 6.8) for 20 min followed by equilibration in alkylation buffer (50 mM Tris- $\mathrm{HCl}, 6 \mathrm{M}$ urea, $2 \%(\mathrm{w} / \mathrm{v})$ SDS, 30\% (v/v) glycerol, $2.5 \%(\mathrm{w} / \mathrm{v}$ ) iodoacetamide, $\mathrm{pH} 6.8$ ) for a further $20 \mathrm{~min}$. The equilibrated strips were placed on homogenous $12 \%$ SDS-PAGE gels and electrophoresed overnight at $100 \mathrm{~V}$ using a Protean Xi-II Cell (Bio-Rad Laboratories). Resulting gels were stained with Coomassie Brilliant Blue $\mathrm{R}$ and scanned using a Typhoon Trio Variable Mode Imager (GE Healthcare, Freiburg, Germany). Three replicate gels of each, treated and untreated were submitted for analysis using the Image Master Platinum software (GE Healthcare, Freiburg, Germany) to identify differentially regulated spots. The protein spots of interest on each gel were detected, normalised, edited and manually matched to a reference gel (Fig. 1A).

\subsection{MALDI-ToF mass spectrometry}

Selected proteins were excised from 2D-PAGE gels using an automated spot cutter (GE Healthcare, Freiburg, Germany), digested with trypsin and analysed as previously described (Carberry et al., 2006). Mass spectrometry was carried out using an Ettan ${ }^{\mathrm{TM}}$ MALDI-ToF mass spectrometer (GE Healthcare, Freiburg, Germany), internal calibrants, Angiotensin III (Sigma-Aldrich) and ACTH fragment 18-39 (Sigma-Aldrich), were used to calibrate all spectra. Protein identification was carried out either by $\mathrm{m} / \mathrm{z}$ data interrogation of a (I) local FASTA version of the annotated A. fumigatus genome available at http://www.cadre-genomes.org.uk/ and (II) $\operatorname{Mascot}^{\mathrm{TM}}$ nrNCBI database (Mabey et al., 2004).

\subsection{GSH/GSSG and superoxide dismutase activity determination}

Intracellular glutathione was measured using the method of Thön et al. (2010) and Rahman et al. (2006), with modifications. A. fumigatus ATCC26933 and $\Delta$ gliT (Schrettl et al., 2010), respectively, were cultured for $21 \mathrm{~h}$ in AMM before addition of gliotoxin ( $5 \mu \mathrm{g} / \mathrm{ml}$ final) for $3 \mathrm{~h}$. Mycelia were harvested through miracloth and dried. Mycelia $(500 \mathrm{mg})$ in $5 \%(\mathrm{w} / \mathrm{v})$ SSA $(500 \mu \mathrm{l})$ were beadbeaten at $30 \mathrm{~Hz}$ for $5 \mathrm{~min}$ followed by centrifugation at $12,000 \mathrm{~g}$ for $10 \mathrm{~min}$ at $4{ }^{\circ} \mathrm{C}$. Supernatants were removed, covered in tinfoil and neutralised using triethanolamine. All samples were diluted $(1 / 10-1 / 30)$ in $125 \mathrm{mM}$ sodium phosphate, $6.3 \mathrm{mM}$ EDTA, pH 7.5 prior to centrifugation at $12,000 \mathrm{~g}$ for $10 \mathrm{~min}$ at $4{ }^{\circ} \mathrm{C}$. Whole cell 


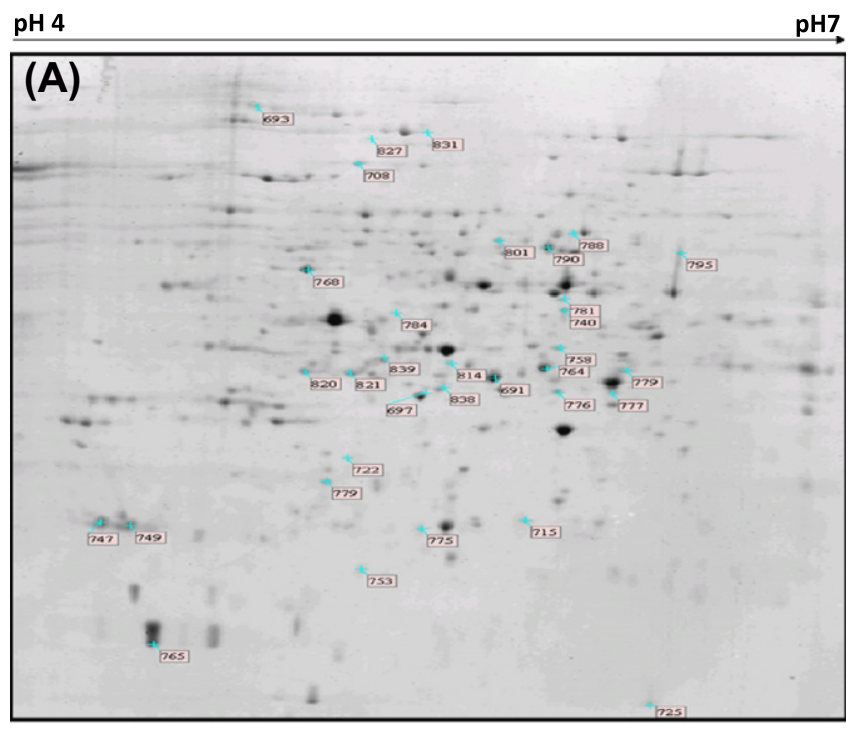

(B)
Gliotoxin $\quad \begin{gathered}\text { Exogenous Gliotoxin } \\ \text { Present }\end{gathered}$
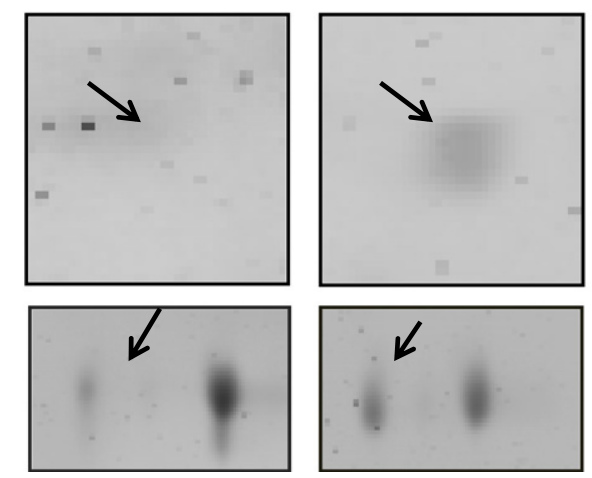

AFUA_5G09240

$\mathrm{Cu}, \mathrm{Zn}$ Superoxide Dismutase

AFUA_6G02280

Allergen Asp f 3

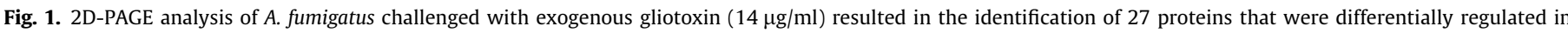

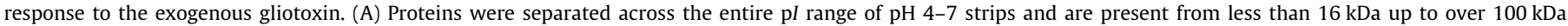

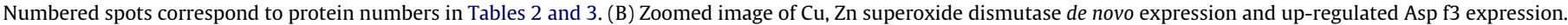

protein was also extracted in the same manner using lysis buffer (100 mM Tris- $\mathrm{HCl}, 50 \mathrm{mM} \mathrm{NaCl}, 20 \mathrm{mM}$ EDTA, 10\% (v/v) glycerol, $30 \mathrm{mM}$ DTT, $1 \mathrm{mM}$ PMSF, and $1 \mu \mathrm{g} / \mathrm{ml}$ pepstatin $\mathrm{A}, \mathrm{pH} 7.5)$ and quantified by Bradford Assay. 2-vinylpyridine ( $2 \mu \mathrm{l}, 1 / 10$ in assay buffer) was added for GSSG determination, incubated at room temperature for $1 \mathrm{~h}$ before triethanolamine $(6 \mu \mathrm{l})$ was added to neutralise the 2-vinylpyridine. All samples were incubated for $10 \mathrm{~min}$ at room temperature prior to assay. Then, blanks/standards/samples $(20 \mu \mathrm{l})$ were added to 96 -well microtiter plates followed by $10 \mathrm{mM}$ dithionitrobenzoic acid (DTNB) containing 1 unit glutathione reductase and left for $30 \mathrm{~s}$. NADPH ( $5 \mathrm{mM})$ was added to each well, microplates gently shaken and A412 nm measured after $90 \mathrm{~s}$. Superoxide dismutase activity was determined in mycelial protein lysates ( $n=3$ each), obtained following mycelial incubation with and without gliotoxin $(14 \mu \mathrm{g} / \mathrm{ml})$ for $4 \mathrm{~h}$. Superoxide dismutase assays were carried out as described by Raimondi et al. (2010), except that nitroblue tetrazolium was used instead of cytochome $c$.

\subsection{Plate assays}

For plate assays, $10^{3}-10^{5}$ freshly harvested conidia of various Aspergillus species, including A. nidulans, A. flavus, A. niger, A. oryzae, A. terreus, Neurospora crassa, Cochliobolus heterostrophus and
Fusarium graminearum were point inoculated on plates containing gliotoxin in a range of $0,0.1-30 \mu \mathrm{g} / \mathrm{ml}$. As controls, A. fumigatus wild-type ATCC46645 and $\Delta g l i T$ (Schrettl et al., 2010) were used. The diameter of the fungal colonies was determined after $72 \mathrm{~h}$ and normalised to the diameter on plates without the addition of gliotoxin. For experiments utilising $S$. cerevisiae, strain background

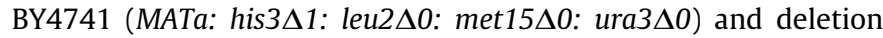
derivatives were used throughout. Yeast strains deleted for SOD1 and GSH1 were purchased from Euroscarf. Spot growth assays were carried out by diluting an overnight culture of cells in fresh YPD medium to $1 \times 10^{6}$ cells $/ \mathrm{ml}$. Cells were then grown to exponential phase to a density of $3 \times 10^{6}$ cells $/ \mathrm{ml}$. Cells were then re-suspended in YPD to a density of $5 \times 10^{6}$ cells $/ \mathrm{ml}$ and transferred to a microtiter plate. Following a series of 1 in 5 dilutions, cells were transferred to YPD plates using a multi-pronged replicator and incubated at $30^{\circ} \mathrm{C}$ for $2-3$ days. GSH1 coding region \pm 500 bp was cloned into pRS315 (LEU2) by gap repair and efficacy of the construct confirmed by sequencing.

\section{9. ctsD deletion in A. fumigatus $\Delta$ gliT}

An aspartic protease is encoded by $A$. fumigatus cts $D$ (AFUA_4G07040; Vickers et al., 2007). To generate A. fumigatus 
$\triangle c t s D$, the bipartite marker technique (Nielsen et al., 2006) was used, with modifications. Briefly, A. fumigatus $\Delta$ gliT $^{46645}$ (Schrettl et al., 2010) was co-transformed with two DNA constructs, each containing an incomplete fragment of the gliotoxin oxidoreductase gene, gliT (Schrettl et al., 2010; Scharf et al., 2010) fused to $1.5 \mathrm{~kb}$, and $1.7 \mathrm{~kb}$ of $c t s D 5^{\prime}$ and $3^{\prime}$ flanking sequences, respectively. These marker fragments shared a 242 bp overlap within the gliT cassette, which served as a potential recombination site during transformation. Two rounds of PCR generated each fragment (Table 1). First, each flanking region was amplified from A. fumigatus ATCC46645 genomic DNA using primer ogliT-1 and ogliT-2 for flanking region A (1.5 kb), and ogliT-3 and ogliT-4 for flanking region B (1.7 kb). After purification, the fragments were digested with RsrII and Psil, respectively. gliT was PCR amplified from A. fumigatus ATCC46645 genomic DNA and inserted into TOPO cloning vector (Invitrogen). gliT selection marker was removed from the TOPO cloning vector following double restriction digest with RsrII and Psil and ligated with the two flanking regions A and B described above. Subsequently, two overlapping fragments were amplified from the ligation products using primers ogliT-5 and ogliT-6 for fragment $\mathrm{C}$ $(2.1 \mathrm{~kb})$ and primers ogliT-7 and ogliT-8 for fragment D (2.2 kb). For generation of $\Delta c t s D$, A. fumigatus $\Delta g l i T^{46645}$ protoplasts were transformed simultaneously with the overlapping fragments $C$ and D, as described by Schrettl et al. (2010). Subsequent selection was performed on Aspergillus Minimal Medium (AMM) with $0.7 \%$ $(\mathrm{w} / \mathrm{v})$ agar including $1 \mathrm{M}$ sucrose as an osmotic stabiliser and containing gliotoxin $(30 \mu \mathrm{g} / \mathrm{ml})$. Southern analyses, to confirm gliT insertion at the ctsD locus was performed using a digoxigenin (DIG)-labelled probe, generated using primers ogliT-5 and ogliT-2, against the 5 '-flanking region of ctsD following AflIII restriction digest of A. fumigatus transformant genomic DNA. Further Southern analyses, via $X b a$ I digestion of $A$. fumigatus transformant genomic DNA, to confirm the loss of ctsD and acquisition of the gliT, were undertaken using DIG-labelled ctsD and gliT probes prepared by PCR using primers ogliT-9 and ogliT-10 for ctsD and ogliT-11 and ogliT-12 for the gliT probe, respectively. RT-PCR analyses were performed to confirm loss of ctsD expression and assessment of gliT expression using primers ogliT-13 and ogliT-14 for ctsD mRNA analysis and ogliT-7 and ogliT-6 for gliT mRNA analysis.

Table 1

Primers used in this study.

\begin{tabular}{|c|c|c|}
\hline Primer & Sequence $5^{\prime}-3^{\prime}$ & Use(s) \\
\hline ogliT-1 & AAAGGCCATGCGAGCTACTA & Flanking region $\mathrm{A}$ \\
\hline ogliT-2 ${ }^{\mathrm{a}}$ & CCCGGTCCGAATCTATGGTCGC & $\begin{array}{l}\text { Flanking region } A \text {, } \\
5^{\prime} \text {-flanking ctsD probe }\end{array}$ \\
\hline ogliT- $3^{b}$ & CCTTATAAGCATTGATGGGTC & Flanking region $\mathrm{B}$ \\
\hline ogliT-4 & СTCTCTATCAGCGGGAAAAC & Flanking region $\mathrm{B}$ \\
\hline ogliT-5 & CGCCAATGTGACAGAGATCA & $\begin{array}{l}\text { Overlapping fragment C, } \\
5^{\prime} \text {-flanking ctsD probe }\end{array}$ \\
\hline ogliT-6 & GACACCCACGGTCTCCTG & $\begin{array}{l}\text { Overlapping fragment C, gliT } \\
\text { RT-PCR }\end{array}$ \\
\hline ogliT-7 & АСТССАССАTCCAGTTCCAG & $\begin{array}{l}\text { Overlapping fragment D, gliT } \\
\text { RT-PCR }\end{array}$ \\
\hline ogliT-8 & AGTCCGGATTCGACGTACTG & Overlapping fragment D \\
\hline ogliT-9 & TTTCTCTCGCCTCCACAGTT & ctsD Probe \\
\hline ogliT-10 & TTGTAGCTAAGGCCCGAGAA & ctsD Probe \\
\hline ogliT-11 & CTACTCTCGCGCATACCACA & gliT Probe \\
\hline ogliT-12 & GCTCCTGATCGAGACGAAAC & gliT Probe \\
\hline ogliT-13 & GAATGGTACGCCTTGTCGTT & ctsD RT-PCR \\
\hline ogliT-14 & TTGTAGCTAAGGCCCGAGAA & ctsD RT-PCR \\
\hline Calm $\mathrm{F}^{\mathrm{c}}$ & CCGAGTACAAGGAAGCTTTCTC & calm RT-PCR \\
\hline \multirow[t]{2}{*}{ Calm $\mathrm{R}^{\mathrm{c}}$} & GAATCATCTCGTCGATTCGTCGT & \\
\hline & CTCAGT & calm RT-PCR \\
\hline
\end{tabular}

\footnotetext{
${ }^{\text {a }}$ RsrII restriction site underlined.

b Psil restriction site underlined.

c Burns et al. (2005).
}

\section{Results}

3.1. Determination of media-dependence of gliotoxin production in A. fumigatus

The concentration of gliotoxin in Sabouraud and MEM/FCS culture supernatants was determined to be $1.35 \pm 0.3 \mu \mathrm{g}$ and $23.65 \pm 1.3 \mu \mathrm{g}$ per mg mycelia, respectively. Thus, a 17 -fold difference in gliotoxin production was evident which was directly related to culture medium composition. Moreover, this observation facilitated evaluation of the effect of increased extracellular gliotoxin levels on A. fumigatus, against a background of minimal gliotoxin biosynthesis and secretion.

\subsection{Exogenous gliotoxin alters specific protein expression in A. fumigatus}

Following exposure of $A$. fumigatus in Sabouraud media ( $24 \mathrm{~h}$ ) to control conditions (methanol) and elevated gliotoxin $(700 \mu \mathrm{g}$ in methanol) addition, mycelia were harvested after $4 \mathrm{~h}$ and lysed, followed by 2D-PAGE, comparative protein expression analysis and MALDI-ToF mass spectrometry to facilitate protein identification (Fig. 1A and B, Tables 2 and 3). Overall, perturbation in the expression of at least 27 distinct proteins was detected, comprising of both up-regulation $(18 ; 64 \%)$ and down-regulation $(9 ; 33 \%)$ in response to gliotoxin exposure. De novo expression of a $\mathrm{Cu}, \mathrm{Zn}$ superoxide dismutase (AFUA_5G09240; SOD; Fig. 1B), a short chain dehydrogenase (AFUA_4G08710) and a eukaryotic translation elongation factor $\beta 1$ subunit (AFUA_1G11190) were also clearly observed following gliotoxin exposure (Table 2). Interestingly, superoxide dismutase activity was significantly elevated $(p<0.03$ ) from 0 (pre-exposure) to $92 \pm 41 \mathrm{U} / \mathrm{mg}$ in cultures exposed to gliotoxin, in accordance with proteomic data. Moreover, reactive oxygen species (ROS) production, also accompanied mycelial exposure to gliotoxin (data not shown). Three proteins (putative adenine phosphoribosyltransferase, uridylate kinase and an aminopeptidase) exhibited between 10-20-fold increased expression, while three proteins: Vip1, a putative thiazole biosynthesis enzyme and allergen Asp f3 (Fig. 1B) were up-regulated 9.49, 7.03 and 5.8-fold, respectively. In addition, expression of eight other proteins, including a putative heat shock protein, Sti1, and thiamine biosynthesis protein, Nmt1, was induced by 1.9-3.0-fold. Overall, identification of proteins whose expression was up-regulated by gliotoxin exposure was confirmed by overall sequence coverage ranging from 17.6-49.3\%. The expression of nine proteins was found to be significantly $(p<0.05)$ down-regulated following gliotoxin exposure (Table 3). Mycelial catalase (AFUA_3G02270) expression reduced by over 40 -fold, while that of ATP-citrate lyase (AFUA_6G10660) and fumaryl acetoacetate hydrolase (FahA; AFUA_2G04230) diminished by over 26 and 21-fold, respectively. The expression of the remaining six proteins, including antioxidant protein LsfA (peroxredoxin; AFUA_4G08580) and dehydrogenases/ oxidoreductases, ranged from a 4.2-9.6-fold reduction, compared to control conditions.

\subsection{Intracellular GSH levels are significantly altered in A. fumigatus AgliT}

GSH levels were significantly elevated $(p<0.05)$ in A. fumigatus $\Delta$ gliT compared to wild-type (Fig. $2 \mathrm{~A}$ ) which suggests that elevated GSH may potentiate the autotoxic effects of gliotoxin. Differences in intracellular GSH levels remained significantly different $(p<0.05)$, in A. fumigatus wild-type and $\Delta$ gliT, even in the presence of gliotoxin $(5 \mu \mathrm{g} / \mathrm{ml})$. However addition of gliotoxin, to either A. fumigatus wild-type or $\Delta g l i T$, resulted in a diminution in intracellular GSH 
Table 2

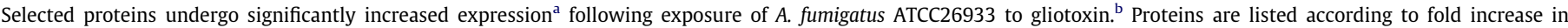
expression.

\begin{tabular}{|c|c|c|c|c|c|}
\hline Proposed/actual function & Fold increase & \% Sequence coverage & Mass (Da) & CADRE I.D. $^{\mathrm{c}}$ & Spot No. \\
\hline $\mathrm{Cu}, \mathrm{Zn}$ superoxide dismutase & $(+)$ Unique & 33.5 & 16,361 & AFUA_5G09240 & 725 \\
\hline Short chain dehydrogenase & (+) Unique & 29.6 & 30,928 & AFUA_4G08710 & 779 \\
\hline \multicolumn{6}{|l|}{ Eukaryotic translation elongation } \\
\hline Factor $1 \beta$ subunit & $(+)$ Unique & 25.9 & 30,142 & AFUA_1G11190 & 714 \\
\hline Adenine phosphoribosyltransferase & 20.5 & 33.6 & 23,621 & AFUA_7G02310 & 715 \\
\hline Uridylate kinase & 10.3 & 33.2 & 25,560 & AFUA_7G03990 & 722 \\
\hline Aminopeptidase & 10.1 & 26 & 106,227 & AFUA_4G09030 & 693 \\
\hline Vip1 protein ${ }^{\mathrm{d}, \mathrm{e}}$ & 9.5 & 24.2 & 28,265 & AFUA_2G10030 & 777 \\
\hline Vip1 protein $^{\mathrm{d}, \mathrm{e}}$ & 7.0 & 44.2 & 28,265 & AFUA_2G10030 & 776 \\
\hline Thiazole biosynthesis enzyme & 7.0 & 27.7 & 35,492 & AFUA_6G08360 & 814 \\
\hline Allergen Asp F3 & 5.8 & 30.4 & 18,453 & AFUA_6G02280 & 765 \\
\hline Nitroreductase family protein & 3.0 & 49.3 & 24,314 & AFUA_5G09910 & 753 \\
\hline Unknown function protein ${ }^{\mathrm{d}, \mathrm{e}}$ & 2.9 & 30.8 & 25,439 & AFUA_5G14680 & 749 \\
\hline Heat shock protein (Sti1), putative & 2.9 & 17.6 & 65,031 & AFUA_7G01860 & 708 \\
\hline Allantoicase & 2.7 & 21.3 & 46,273 & AFUA_3G12560 & 740 \\
\hline \multicolumn{6}{|l|}{ Mitochondrial processing } \\
\hline Peptidase $\beta$ subunit & 2.7 & 31.5 & 53,270 & AFUA_1G14200 & 768 \\
\hline Glycerol dehydrogenase & 2.5 & 25.8 & 36,828 & AFUA_4G11730 & 691 \\
\hline Unknown function protein ${ }^{\mathrm{d}, \mathrm{e}}$ & 2.1 & 37.9 & 25,439 & AFUA_5G14680 & 747 \\
\hline Thiamine biosynthesis protein Nmt1 & 1.9 & 33.9 & 38,323 & AFUA_5G02470 & 764 \\
\hline Phosphoglycerate kinase & 1.9 & 34.2 & 44,761 & AFUA_1G10350 & 758 \\
\hline
\end{tabular}

a $p<0.05$.

b GliT (AFUA_6G09740) expression was also significantly increased, 2.5-3.5-fold (Schrettl et al., 2010).

c CADRE I.D: CADRE Identification (Mabey et al., 2004).

d,e Vip1 protein and unknown function protein were each detected in two adjacent protein spots, respectively.

Table 3

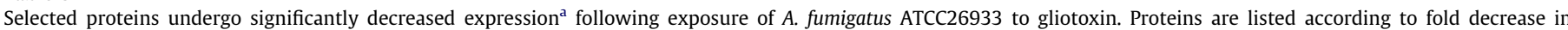
expression.

\begin{tabular}{|c|c|c|c|c|c|}
\hline Proposed/actual function & Fold decrease & \% Sequence coverage & Mass (Da) & CADRE I.D. ${ }^{\mathrm{b}}$ & Spot No. \\
\hline Mycelial catalase 1 & -41.6 & 20.3 & 79,910 & AFUA_3G02270 & 827 \\
\hline ATP-citrate lyase & -26.2 & 30.7 & 52,918 & AFUA_6G10660 & 790 \\
\hline Fumarylacetoacetate hydrolase FahA & -21.2 & 18.3 & 46,817 & AFUA_2G04230 & 781 \\
\hline Aminopeptidase B & -9.6 & 7.9 & 108,707 & AFUA_5G04330 & 831 \\
\hline Aldo-keto reductase family 7 protein & -9.0 & 37.2 & 35,489 & AFUA_5G02020 & 697 \\
\hline Aldehyde dehydrogenase & -8.9 & 25.6 & 53,759 & AFUA_7G01000 & 795 \\
\hline Alcohol dehydrogenase, $\mathrm{Zn}$ containing & -8.5 & 47.1 & 37,854 & AFUA_1G04620 & 779 \\
\hline Anti-oxidant protein LsfA peroxiredoxin & -6.9 & 31.9 & 23,393 & AFUA_4G08580 & 775 \\
\hline GMC oxidoreductase & -4.2 & 17.6 & 72,151 & AFUA_3G01580 & 706 \\
\hline
\end{tabular}

a $p<0.05$.

b CADRE I.D, CADRE identification (Mabey et al., 2004).

levels (nmol/mg protein), in both strains, without a concomitant increase in GSSG. Although not statistically significant, these trends were reproducible, and suggest formation of mixed gliotoxin-GSH disulphides. Moreover, the GSH/GSSG ratio in $A$. fumigatus ATCC26933 was 8.15, while it was increased to 47.5 in A. fumigatus $\Delta$ gliT. This dramatic increase in ratio in A. fumigatus $\Delta$ gliT must be caused by the significant increase $(p<0.05)$ in GSH levels as there was no observed difference in the GSSG levels. Relatedly, A. fumigatus $\Delta g l i T$ was significantly more resistant to the effects of diamide, a GSH scavenger, than either wild-type or gliT ${ }^{C}$ strains (Fig. 2C), thereby providing complementary support for the observed GSH levels. Future work will investigate the effects of gliotoxin on GSH reductase and peroxidase activities, respectively, to further elucidate the interplay between GliT, gliotoxin and these catalytic functions.

\subsection{Gliotoxin disrupts the intracellular redox balance in S. cerevisiae}

Fig. 3A shows that growth of $S$. cerevisiae BY4741 (wild-type) is impeded by exposure to exogenous gliotoxin $(8 \mu \mathrm{g} / \mathrm{ml})$ and that $S$. cerevisiae $\Delta$ sod1 and $\Delta y a p 1$ exhibit increased hypersensitivity to exogenous gliotoxin compared to the wild-type strain. This observation indicates that gliotoxin induced an oxidative stress in $S$. cerevisiae and may cause superoxide formation. Importantly, compared to wild-type, no growth difference was observed when S. cerevisiae $\Delta c t t 1, \Delta g s h 2$ or $\Delta g l r 1$ were exposed to identical gliotoxin concentrations (data not shown). To further explore the mechanism underlying gliotoxin-mediated growth inhibition, $S$. cerevisiae $\Delta$ gsh1, which is deficient in intracellular GSH (Lee et al., 2001), and $\Delta g s h 1: G S H 1$, the complemented strain, were exposed to gliotoxin. It can be seen in Fig. $3 \mathrm{~B}$ that $S$. cerevisiae $\Delta g$ sh1 exhibited increased resistance to exogenous gliotoxin $(16 \mu \mathrm{g} / \mathrm{ml})$ compared to wild-type and that re-introduction of GSH1 in the deletion strain restored gliotoxin sensitivity. This observation suggests that decreased intracellular GSH levels may protect $S$. cerevisiae against gliotoxin by attenuating production of intracellular reduced gliotoxin (gliotoxin- $(\mathrm{SH})_{2}$ ).

\subsection{Gliotoxin inhibits fungal growth}

The proposed use of gliT as a selection marker during transformation in fungi relies on the sensitivity of these fungal species to gliotoxin (Schrettl et al., 2010). Hence, it was necessary to test the susceptibility of potential target fungi towards treatment with 

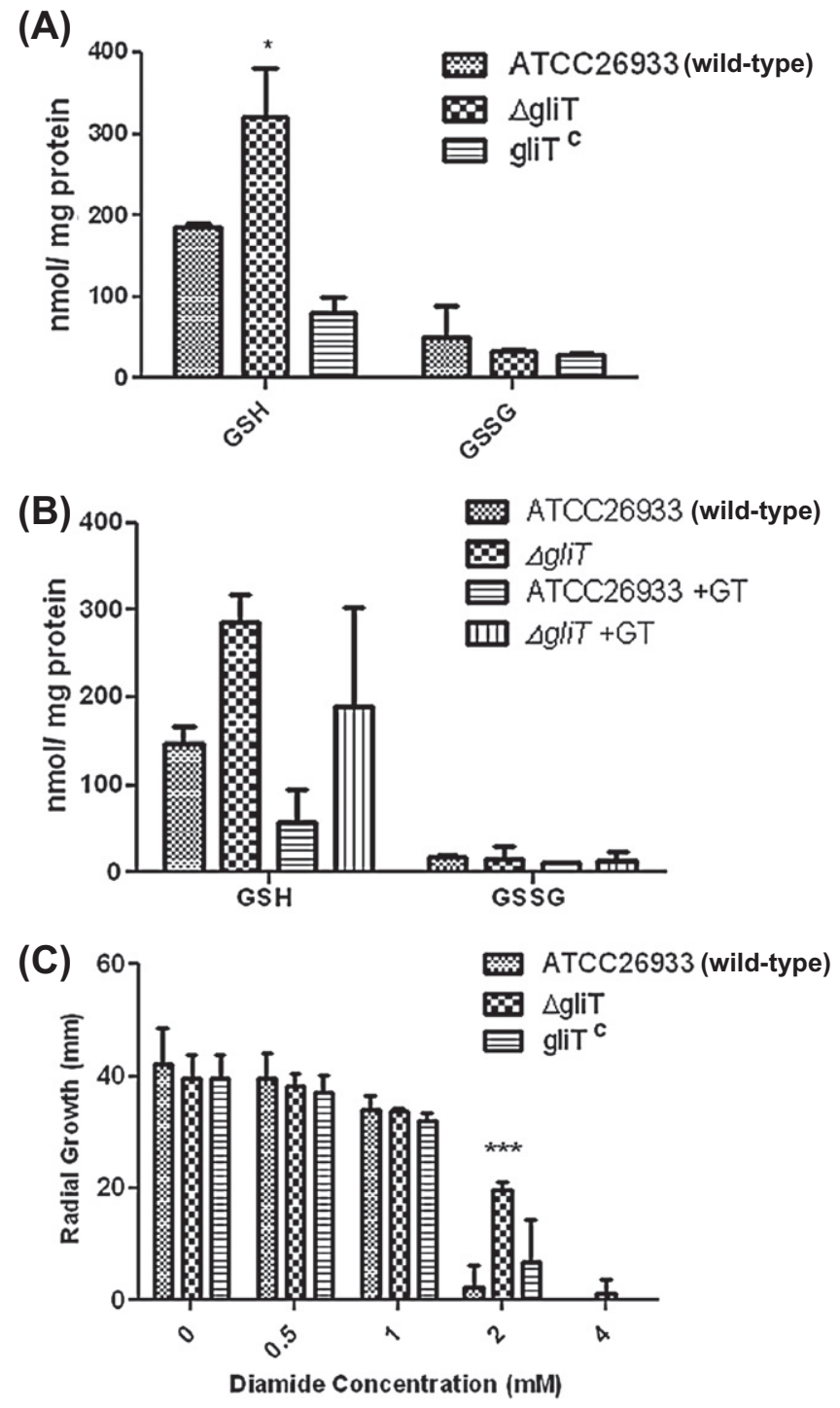

Fig. 2. Assessment of redox status in A. fumigatus $\Delta g l i T$. (A) Significantly elevated GSH is evident in A. fumigatus $\Delta$ gliT compared to wild-type and A. fumigatus gliT. This may sensitise the mutant to gliotoxin. (B) GSH and GSSG levels in A. fumigatus ATCC26933 and $\Delta$ gliT in the absence and presence of gliotoxin (GT) for $3 \mathrm{~h}$. Under basal conditions, there is a significant increase $(p<0.05)$ in GSH levels in $A$. fumigatus $\Delta$ gliT compared to A. fumigatus ATCC26933. Gliotoxin addition depletes GSH in both strains without affecting the significant difference $(p<0.05)$ between GSH levels in both strains. (C) A. fumigatus $\Delta g l i T$ is significantly more resistant to the GSH-scavenging agent, diamide. This is most likely a consequence of elevated intracellular GSH. In both cases, complementation restored the wild-type phenotype.

gliotoxin. This was achieved by performing plate assays containing different concentrations of gliotoxin. For plate assays, $10^{3}-10^{5}$ freshly harvested conidia of various Aspergillus species, including A. nidulans, A. flavus, A. niger, A. oryzae, A. terreus, $N$. crassa, C. heterostrophus and $F$. graminearum were point inoculated on plates containing gliotoxin in a range of $0,0.1-30 \mu \mathrm{g} / \mathrm{ml}$. Statistically significant ( $p<0.001$ using Bonferronis Multiple Comparison Test), gliotoxin-mediated growth inhibtion of A. nidulans, A. terreus, A. niger, $C$. heterostrophus and $N$. crassa was observed at $5 \mu \mathrm{g} / \mathrm{ml}$ gliotoxin (Fig. 4A and B). This establishes all of these strains as suitable for transformation with gliT to facilitate transformant selection on gliotoxin. Growth of A. flavus, F. graminarium and $A$. oryzae was significantly inhibited $(p<0.001)$ at $10 \mu \mathrm{g} / \mathrm{ml}$ gliotoxin (Fig. 4). Again, this indicates suitability of these as target strains for transformation with gliT to facilitate transformant selection on gliotoxin, albeit necessitating the use of higher concentrations of gliotoxin to facilitate selection.

3.6. Targeted gene deletion in A. fumigatus can be detected by recovery of gliotoxin resistance

A. fumigatus ATCC46645 ctsD (AFUA_4G07040) encodes an aspartic protease which was found to be expressed in minimal and proteinaceous media only, and does not play any role in gliotoxin metabolism (Vickers et al., 2007; Gardiner and Howlett, 2005). After confirmation of $c t s D$ expression in gliotoxin-sensitive A. fumigatus $\Delta$ gli $^{46645}$, targeted deletion of $c t s D$ was undertaken using a bipartite strategy (Nielsen et al., 2006) whereby gliT introduction at the ctsD locus (Fig. 5A), followed by selection of transformants on gliotoxin-containing media $(30 \mu \mathrm{g} / \mathrm{ml})$ was attempted (Fig. 5B). Southern blot analysis of A. fumigatus ATCC 46645 or $\Delta$ gli $T^{46645}$, using a probe for the $5^{\prime}$ flanking region of $c t s D$, yielded a fragment size of $3530 \mathrm{bp}$ at the $c t s D$ locus, whereas transformants $(n=10)$ which had recovered gliotoxin resistance yielded a fragment at 2508 bp thereby confirming disruption of $c t s D$. Furthermore, a fragment of $4832 \mathrm{bp}$, which contained the $c t s D$ open reading frame was absent in all 10 transformants when a ctsD coding region probe was employed for Southern analysis (Fig. 5C). Concomitant re-introduction of the gliT coding sequence at the $c t s D$ locus, detected using a gliT-specific probe, in A. fumigatus $\Delta g l i T$, was evident as shown by appearance of a fragment at $5102 \mathrm{bp}$ (corresponding signals for A. fumigatus ATCC 46645 and $\Delta g_{l i T}{ }^{46645}$, were $6403 \mathrm{bp}$ and no signal, respectively) (Fig. 5D). Both gliT and ctsD expression was evident in A. fumigatus ATCC 46645, however as assessed by RT-PCR, ctsD expression was not evident in A. fumigatus $\Delta c t s D^{46645}$ but was detectable in A. fumigatus $\Delta$ gli $T^{46645}$ (Fig. 5E). Conversely, gliT was expressed in A. fumigatus $\Delta c t s D^{46645}$ which confirmed that successful ctsD deletion using gliT re-introduction into A. fumigatus $\Delta g l i T^{46645}$, with selection for gliotoxin resistance, had occurred (Fig. 5E).

\section{Discussion}

Little is known about the effect of gliotoxin on, or in, fungi. Analysis of A. fumigatus culture supernatants revealed gliotoxin production to be media specific, with a 17-fold concentration difference between two specific culture media. 2D-PAGE analysis of A. fumigatus mycelia challenged with exogenous gliotoxin for $4 \mathrm{~h}$ after $24 \mathrm{~h}$ growth in Sabouraud media (low endogenous gliotoxin production) led to the identification of 27 differentially-regulated proteins, 18 which exhibited increased expression, and 9 which underwent decreased expression in the presence of gliotoxin. Only proteins with a $t$-test score of $p \leqslant 0.05$ were accepted as significant. Our data further demonstrates dysregulation of intracellular GSH levels in the absence of A. fumigatus gliT, a possible consequence of which is elevated sensitivity to exogenous gliotoxin. Because of the increased sensitivity or resistance of $S$. cerevisiae gene deletants to gliotoxin, along with sensitivity of a range of fungal species to gliotoxin, we suggest gliotoxin exposure as a standard phenotypic screen in functional genomic studies. Whether gliotoxin is primarily an antifungal, or a protective molecular species, is discussed below - along with the potential for gliT/gliotoxin as an alternative selection marker system for fungal transformation.

$\mathrm{Cu}, \mathrm{Zn}$ superoxide dismutase (Cu, Zn SOD), (AFUA_5G09240) was expressed de novo in the presence of exogenous gliotoxin in A. fumigatus. Interestingly, gliotoxin has been shown to produce superoxide radicals in mast cells (Niide et al., 2006), and the de novo expression of $\mathrm{Cu}, \mathrm{Zn}$ SOD suggests that gliotoxin addition may have an identical effect in A. fumigatus. Moreover, $\mathrm{Cu}, \mathrm{Zn}$ SOD was previously identified by Lessing et al. (2007), to be 
(A) Gliotoxin $(0 \mu \mathrm{g} / \mathrm{ml})$

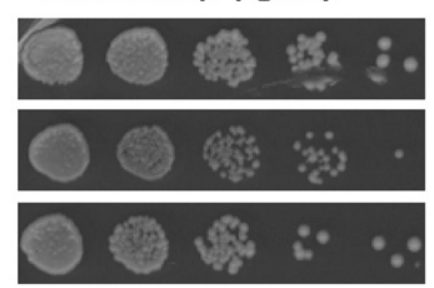

(B) Gliotoxin $(0 \mu \mathrm{g} / \mathrm{ml})$

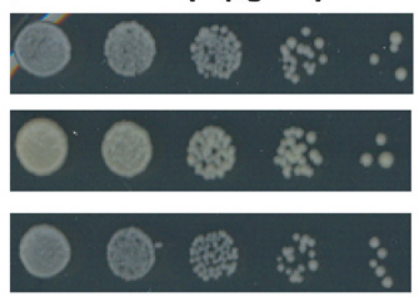

BY4741 (WT)

$\Delta \operatorname{sod} 1$

$\triangle$ yap1

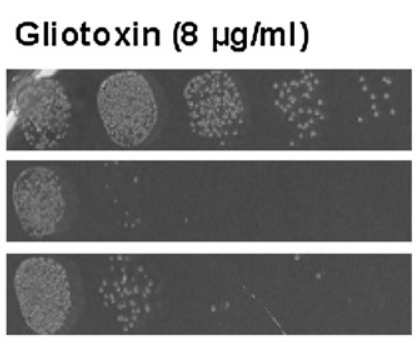

Gliotoxin $(16 \mu \mathrm{g} / \mathrm{ml})$

BY4741 (WT)

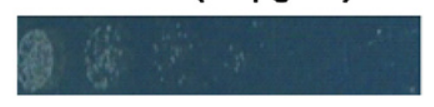

$\Delta g s h 1$

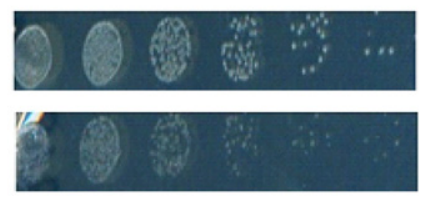

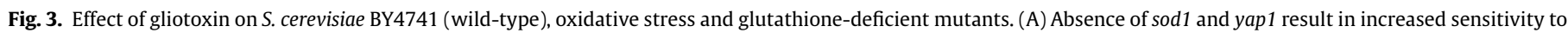
exogenous gliotoxin. (B) S. cerevisiae sensitivity to gliotoxin is dependent on GSH1 presence.

involved in defence against oxidative radicals in A. fumigatus. Thus, although not essential for virulence (Lambou et al., 2010), the enzyme appears to play a role in ROS and protection against gliotoxin. Interestingly, SOD expression was also increased in A. flavus during the production of another mycotoxin, aflatoxin (O'Brian et al., 2007) and production of aflatoxin has been linked with greater oxygen requirements and high levels of ROS (Narasaiah et al., 2006) as has also been suggested for gliotoxin (Watanabe et al., 2004). A. fumigatus SOD has been shown to be reactive to sera from aspergillosis patients (Holdom et al., 1996), and is present on the cell wall and in culture media. This may show a possible protective role against the oxidative burst mechanism of neutrophils in response to A. fumigatus infection (Hamilton et al., 1996). Bruns et al. (2010) also noted that $\mathrm{Cu}, \mathrm{Zn}$ SOD gene expression was significantly up-regulated in A. fumigatus during biofilm formation. De novo expression of eukaryotic elongation factor $1 \beta$ subunit (AFUA_1G11190) was also observed in A. fumigatus mycelia. This protein was previously identified to be abundant in A. fumigatus conidia but was found to be relatively weakly immunogenic (Asif et al., 2006). Sugui et al. (2008) demonstrated that short chain dehydrogenase expression (AFUA_4G08710) was significantly upregulated in conidia, but not hyphae, upon exposure to neutrophils from either normal or CGD donors. Thus, the de novo expression of this protein in response to gliotoxin exposure may be due similarities between the intracellular oxidative stress program induced by neutrophils and gliotoxin, respectively. Significantly, cognate mRNA expression of six (adenine phosphoribosyltransferase (APRT), aminopeptidase, Vip1 protein, heat shock protein (Sti1), glycerol dehydrogenase (GldB) and thiamine biosynthesis protein (Nmt1); Table 2) of the 15 proteins whose expression is induced by gliotoxin, was also significantly up-regulated following conidial exposure to airway epithelial cells (Oosthuizen et al., 2011). Elevated expression of two enzymes involved in nucleotide biosynthesis (APRT and uridylate kinase) was also evident upon gliotoxin exposure. Khalaj et al. (2011) noted a 20\% reduction in uridylate kinase expression in an annexin disruptant ( $\Delta a n x C 4)$ and although no functional role was assigned to altered uridylate kinase expression, these authors noted that alterations in stress response related proteins suggested an oxidative stress response in the mutant. Allergen Asp f3 (AFUA_6G02280) is a thiol-specific peroxiredoxin that reduces hydroperoxides to protect against oxidative damage (Fig. 1B). It is a known fungal allergen, is identical to peroxisomal membrane protein Pmp20 and, as a vaccine, has recently been demonstrated to induce cell-mediated protection against Aspergillus infection (Mabey et al., 2004; Diaz-Arevalo et al., 2011). Pmp20 characterisation in Candida boidinii suggested that it functioned to degrade ROS generated at peroxisomal membrane surface, such as lipid hydroperoxides (Horiguchi et al., 2001).

Interestingly, expression of several stress-induced proteins was decreased in the presence of exogenous gliotoxin including mycelial catalase and antioxidant protein, peroxiredoxin LsfA. Mycelial catalase (AFUA_3G02270) expression decreased nearly 42-fold in expression. Lessing et al. (2007) have shown that hydrogen peroxide exposure down-regulates expression of a distinct catalase (AFUA_2G00200) but did not mention AFUA_3G02270. Nonetheless, their observation is in accordance with our findings that oxidative stress conditions may, unexpectedly, suppress catalase levels. Recently, gliotoxin was identified as an antioxidant in mammalian cells, functioning as a peroxiredoxin in the thioredoxin redox system (Choi et al., 2007). Consequently, we speculate that exogenous gliotoxin may fulfil this biological function in A. fumigatus, resulting in diminished requirement for mycelial catalase. Peroxiredoxin LsfA (AFUA_4G08580) is a putative alkyl hyderoperoxide reductase/thiol specific antioxidant, and as such, may have several predicted functions such as antioxidant and oxidoreductase activities. In this case, it appears that gliotoxin exposure down-regulates, LsfA levels (approximately seven-fold), while $\mathrm{H}_{2} \mathrm{O}_{2}$ presence induces expression (Lessing et al., 2007). Overall, the differential regulation of 27 proteins (including one protein of unknown function, AFUA_5G14680) in response to exogenous gliotoxin suggests a hitherto unknown complex interaction between gliotoxin and $A$. fumigatus.

Increased expression (2.5-3.5-fold) of GliT (gliotoxin oxidoreductase; AFUA_6G09740), a component of the gliotoxin biosynthetic cluster was previously reported by us (Schrettl et al., 2010). Interestingly, Schrettl et al. (2008) also observed independent regulation of gliT during comparative transcriptomics study of A. fumigatus wild-type and a mutant deficient in control of siderophore biosynthesis $(\Delta s r e A)$. Moreover, Bruns et al. (2010) detected increased $A$. fumigatus GliT expression during biofilm formation using a proteomic strategy, along with the detection of additional gli genes by transcriptomic analyses. Schrettl et al. (2010) suggested that gliotoxin addition to fungal cultures resulted in GSH depletion, and the data presented herein (Fig. 2B) confirm 


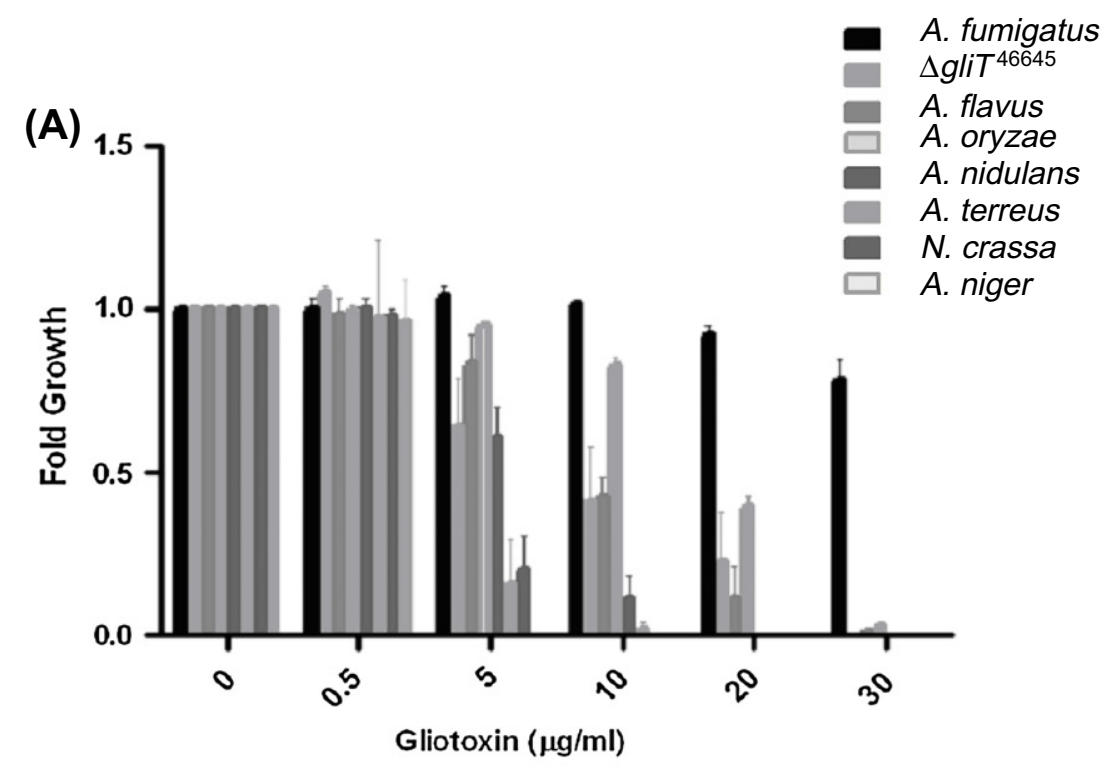

(B)
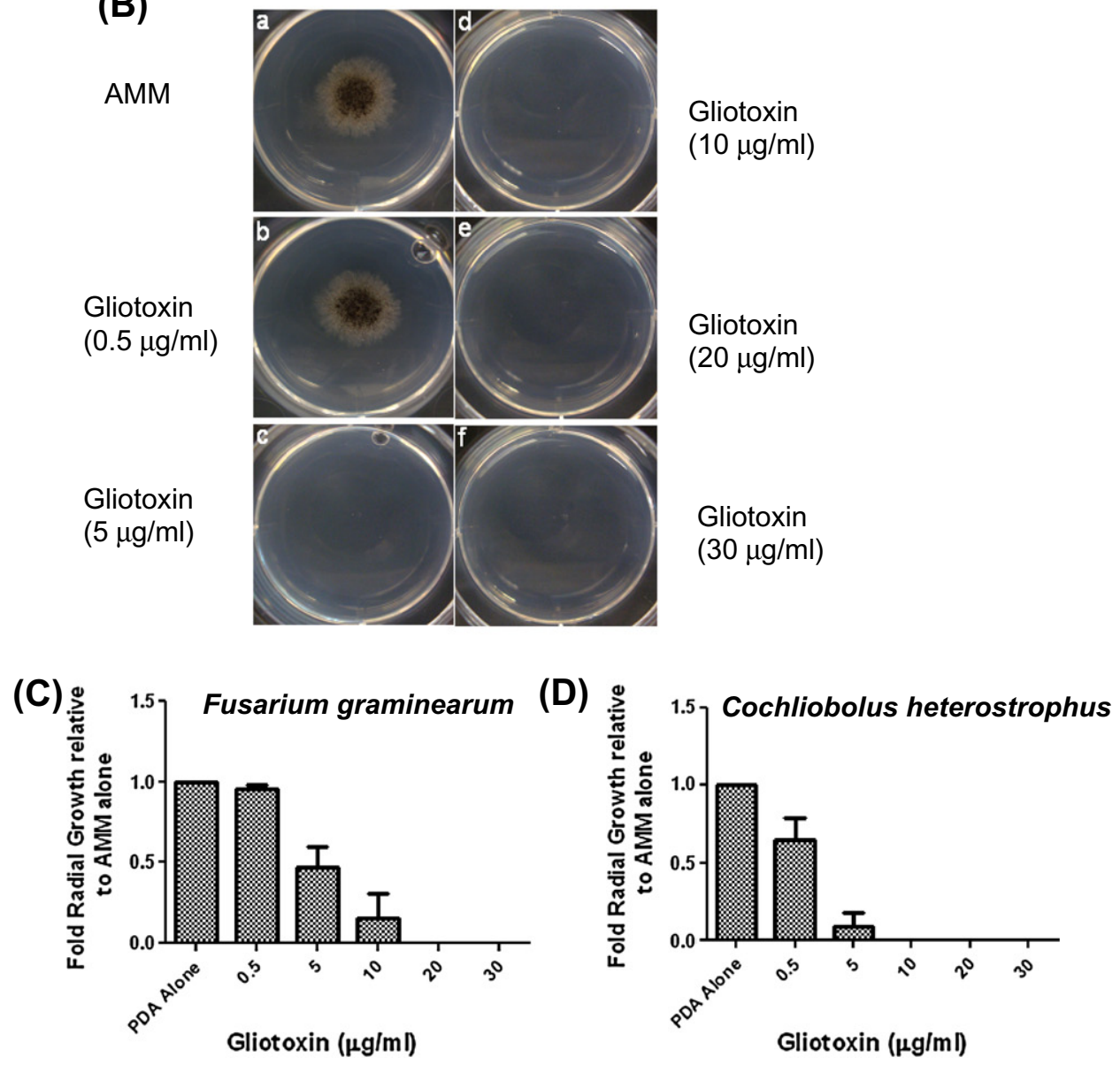

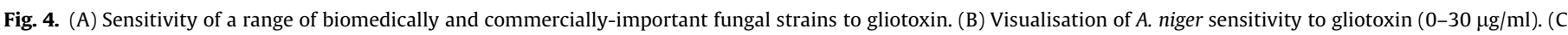

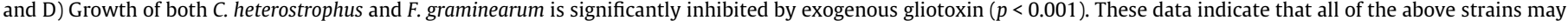
be suitable for use with gliT/gliotoxin selection marker system for both gene deletion studies and heterologous protein expression in fungi.

this prediction. However, we find that deletion of gliT results in significant elevation of intracellular GSH, with concomitant resistance to diamide, compared to A. fumigatus wild-type. This observation suggests that elevated intracellular GSH may actually potentiate the action of gliotoxin on A. fumigatus $\Delta g l i T$, yet simultaneously confer increased resistance towards the GSH-scavanging oxidant, diamide. Interestingly, A. fumigatus $\Delta$ gliT did not exhibit increased sensitivity to $\mathrm{H}_{2} \mathrm{O}_{2}$ (Schrettl et al., 2010), which suggests distinct patterns of redox stress in A. fumigatus. In this regard, Lessing et al. (2007) and Qiao et al. (2008) observed significantly increased sensitivity against $\mathrm{H}_{2} \mathrm{O}_{2}$ and menadione, but not against diamide, in A. fumigatus $\Delta y a p 1$. 
(A)

(i)

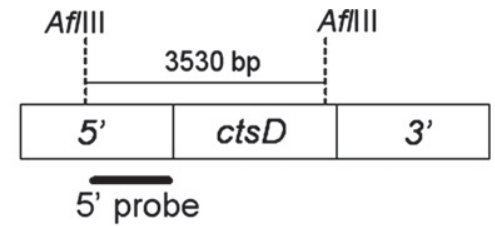

(iii)

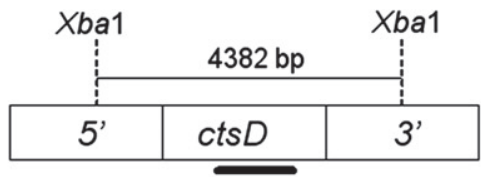

ctsD ORF probe (ii)

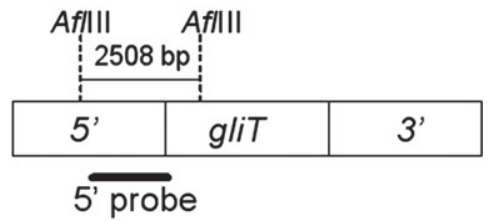

(iv)

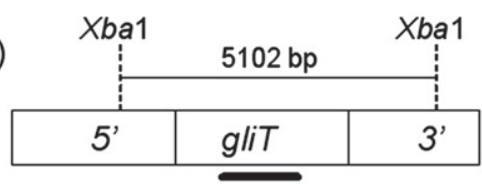

gliT ORF probe

(B)

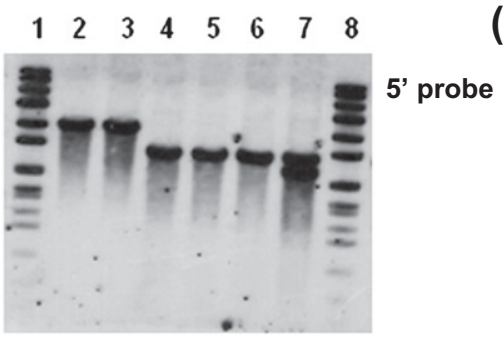

(C)

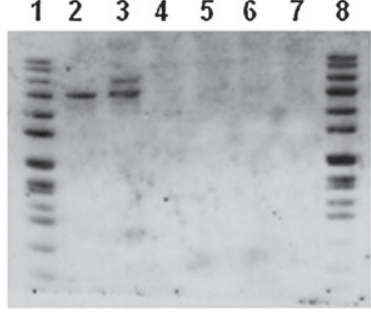

$c t s D$ ORF probe
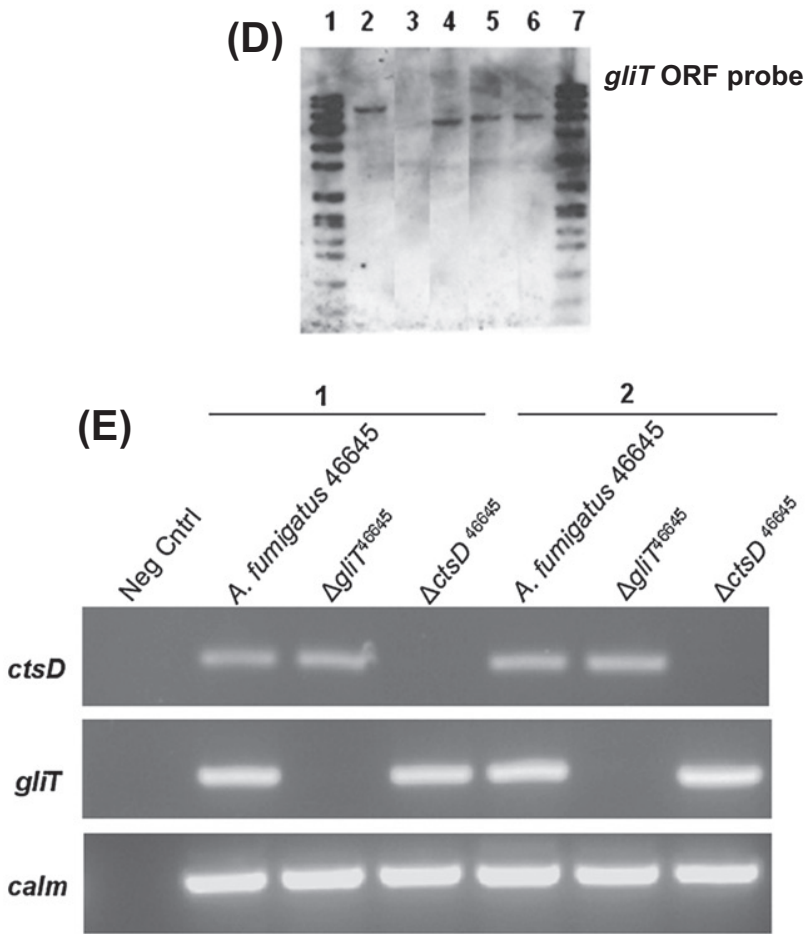

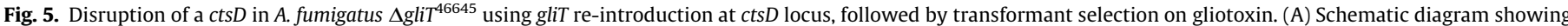

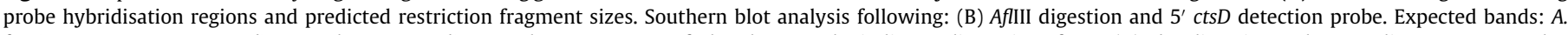

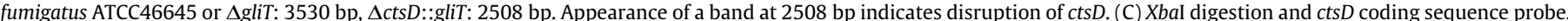

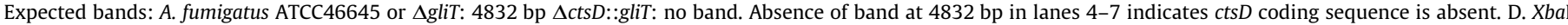

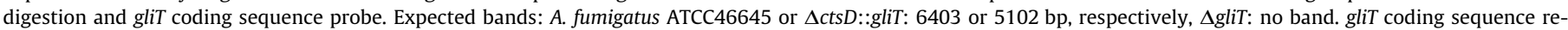

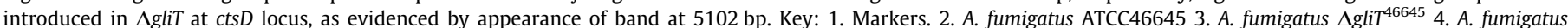

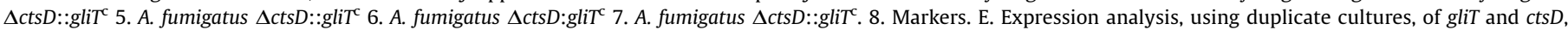

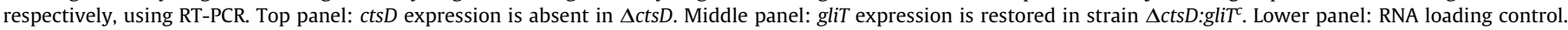

Gliotoxin $(8 \mu \mathrm{g} / \mathrm{ml})$ also adversely affects $S$. cerevisiae whereby $\Delta$ sod1 and $\Delta y a p 1$ growth, respectively, is inhibited to a greater extent than BY4741 (wild-type). This increased sensitivity of $S$. cerevisiae $\Delta$ sod 1 is in accordance with our observation that gliotoxin up-regulates $\mathrm{Cu}, \mathrm{Zn}$ SOD expression in A. fumigatus and predicts superoxide production is consequent to gliotoxin addition in both fungal species. Moreover, the increased sensitivity to gliotoxin in $S$. cerevisiae $\Delta y a p 1$ implies that this gene is also essential to protect against gliotoxin-induced ROS. These data further the observations of Chamilos et al. (2008) regarding the effects of gliotoxin on yeast 
mutants and future studies will explore the effects of gliotoxin on A. fumigatus $\Delta y a p 1$. Our observation that $S$. cerevisiae $\Delta g s h 1$, severely deficient in GSH (Lee et al., 2001), exhibits greater resistance to gliotoxin than wild-type - and that complementation with GSH1 restores wild-type levels of gliotoxin sensitivity - further underpins our proposal that intracellular GSH levels play an important role in mediating gliotoxin cytotoxicity in fungi as has been noted in animals cells by Bernardo et al. (2003).

Gliotoxin was originally investigated as an antifungal antibiotic (Johnson et al., 1943). Later, Losada et al. (2009) investigated the presence of antifungal agents produced by Aspergillus spp and detected gliotoxin in fungal co-culture extracts by LC-MS analysis. Yet, the dramatic sensitivity to gliotoxin $(10 \mu \mathrm{g} / \mathrm{ml} ; 30 \mu \mathrm{M})$ evident in the present work, whereby growth of three fungal species (A. niger, $N$. crassa and C. heterostrophus) was completed inhibited, was unexpected. Notably, all three strains lack the gli cluster and do not possess a gliT ortholog which, at least in part, explains this dramatic gliotoxin sensitivity compared to fungi which possess the cluster with multiple copies of gliT (A. flavus) (Patron et al. 2007; Fox and Howlett, 2008). Coleman et al. (2011) noted the sensitivity of Candida albicans and Cryptococcus neoformans to gliotoxin and A. fumigatus culture supernatants (containing gliotoxin), and speculate about the role of gliotoxin as a competitive factor during host colonisation by multiple fungal species. However, an alternative hypothesis could be that gliotoxin secretion represents a component of the defensive armoury of A. fumigatus against host oxidants, and that the antifungal activity is either accidental, or of more relevance in external environments to facilitate niche competition. Interestingly, Bruns et al. (2010) noted elevated levels of gliotoxin in A. fumigatus biofilms, compared to culture supernatants, and note that this may represent an immunomodulatory strategy (Nishida et al., 2005) on behalf of the organism. However, a more specific interpretation is that a localised gliotoxin "corona" acts externally to dissipate host ROS. If this hypothesis is true, then $A$. fumigatus would have to be able to withstand high local gliotoxin concentrations, which can, in part, explain the protective proteomic remodelling observed herein in response to exogenous gliotoxin addition.

Gliotoxin sensitivity offers the possibility of using gliT as a dominant selection marker for fungal transformation. Consequently, the successful deletion of ctsD (Vickers et al., 2007) from A. fumigatus $\Delta$ gliT, using gliT reconstitution and selection on gliotoxin proves this concept- at least in A. fumigatus. Further refinements of this system, which involves use of a fungal-specific gene (gliT), will involve use of self-excision mechanisms (Hartmann et al., 2010) to release ptrA originally used for gliT deletion (Schrettl et al. 2010) and investigation of the system in commercially important strains (e.g., Trichoderma reesei; Steiger et al., 2011).

\section{Conclusion}

We have demonstrated that gliotoxin addition perturbs the proteome of A. fumigatus and induces de novo $\mathrm{Cu}, \mathrm{Zn}$ SOD expression. Combined with observations of significantly elevated GSH levels in A. fumigatus $\Delta$ gliT and the effects of gliotoxin on S. cerevisiae, we conclude that gliotoxin induces redox stress in fungi, at least in part mediated by superoxide anions and elevated GSH levels. While the antifungal effects of gliotoxin are clear, our results are also in accordance with a complex self-protection system, operative in A. fumigatus. Based on our observations, along with those of Schrettl et al. (2010), Watanabe et al. (2004), Bruns et al. (2010) and Coleman et al. (2011), we now hypothesise that this system may be necessary to defend against high, localised gliotoxin levels (Bruns et al., 2010), present during host colonisation- one function of which may be external dismutation of host ROS. Finally, we demonstrate extreme gliotoxin sensitivity in a range of fungi, the utility of gliT/gliotoxin for targeted gene deletion studies in $A$. fumigatus, and suggest that gliotoxin sensitivity assays should be incorporated into future functional genomic and proteomic studies involving fungi.

\section{Acknowledgments}

S.C. was funded by the Higher Education Authority (HEA) PRTLI Cycles 3 and 4. E.M. was funded by Enterprise Ireland (PC/2008/ 046). S.H. and G.O.K. are Embark Ph.D. Scholars funded by the Irish Research Council for Science Engineering and Technology. HPLC facilities were funded by the HEA and MALDI-ToF MS was funded by the Irish Health Research Board. The assistance of Dr. Joanne Keenan (Dublin City University) with image analysis is gratefully appreciated.

\section{References}

Asif, A.R., Oellerich, M., Amstrong, V.W., Riemenschneider, B., Monod, M., Reichard, U., 2006. Proteome of conidial surface associated proteins of Aspergillus fumigatus reflecting potential vaccine candidates and allergens. J. Proteome Res. 5, 954-962.

Ben-Ami, R., Lewis, R.E., Leventakos, K., Kontoyiannis, D.P., 2009. Aspergillus fumigatus inhibits angiogenesis through the production of gliotoxin and other secondary metabolites. Blood 114, 5393-5399.

Bernardo, P.H., Brasch, N., Chai, C.L., Waring, P., 2003. A novel redox mechanism for the glutathione-dependent reversible uptake of a fungal toxin in cells. J. Biol. Chem. 278, 46549-46555.

Bok, J.W., Chung, D., Balajee, S.A., Marr, K.A., Andes, D., Nielsen, K.F., Frisvad, J.C., Kirby, K.A., Keller, N.P., 2006. GliZ, a transcriptional regulator of gliotoxin biosynthesis, contributes to Aspergillus fumigatus virulence. Infect. Immun. 4, 6761-6768.

Bruns, S., Seidler, M., Albrecht, D., Salvenmoser, S., Remme, N., Hertweck, C., Brakhage, A.A., Kniemeyer, O., Müller, F.M., 2010. Functional genomic profiling of Aspergillus fumigatus biofilm reveals enhanced production of the mycotoxin gliotoxin. Proteomics 10, 3097-3107.

Burns, C., Geraghty, R., Neville, C., Murphy, A., Kavanagh, K., Doyle, S., 2005. Identification, cloning, and functional expression of three glutathione transferase genes from Aspergillus fumigatus. Fungal Genet. Biol. 42, 319-327.

Carberry, S., Neville, C.M., Kavanagh, K.A., Doyle, S., 2006. Analysis of major intracellular proteins of Aspergillus fumigatus by MALDI mass spectrometry: identification and characterisation of an elongation factor 1B protein with glutathione transferase activity. Biochem. Biophys. Res. Commun. 341, 1096-1104.

Chamilos, G., Lewis, R.E., Lamaris, G.A., Albert, N.D., Kontoyiannis, D.P., 2008. Genomewide screening for genes associated with gliotoxin resistance and sensitivity in Saccharomyces cerevisiae. Antimicrobiol. Agents Chemother. 52, 1325-1329.

Choi, H.S., Shim, J.S., Kim, J.A., Kang, S.W., Kwon, H.J., 2007. Discovery of gliotoxin as a new small molecule targeting thioredoxin redox system. Biochem. Biophys. Res. Commun. 359, 523-528.

Coleman, J.J., Ghosh, S., Okoli, I., Mylonakis, E., 2011. Antifungal activity of microbial secondary metabolites. PLoS One 6, e25321.

Cramer Jr., R.A., Gamcsik, M.P., Brooking, R.M., Najvar, L.K., Kirkpatrick, W.R., Patterson, T.F., Balibar, C.J., Graybill, J.R., Perfect, J.R., Abraham, S.N., et al., 2006. Disruption of a nonribosomal peptide synthetase in Aspergillus fumigatus eliminates gliotoxin production. Eukaryot. Cell 5, 972-980.

Davis, C., Carberry, S., Schrettl, M., Singh, I., Stephens, J.C., Barry, S.M., Kavanagh, K., Challis, G.L., Brougham, D., Doyle, S., 2011. The role of glutathione S-transferase GliG in gliotoxin biosynthesis in Aspergillus fumigatus. Chem. Biol. 18 (4), 542552.

Diaz-Arevalo, D., Bagramyan, K., Hong, T.B., Ito, J.I., Kalkum, M., 2011. CD4(+) T cells mediate the protective effect of the recombinant Asp f3-based anti-aspergillosis vaccine. Infect Immun. 79, 2257-2266.

Fox, E.M., Howlett, B.J., 2008. Biosynthetic gene clusters for epipolythiodioxopiperazines in filamentous fungi. Mycol. Res. 112, 162-169.

Gardiner, D.M., Howlett, B.J., 2005. Bioinformatic and expression analysis of the putative gliotoxin biosynthetic gene cluster of Aspergillus fumigatus. FEMS Microbiol. Lett. 248, 241-248.

Gardiner, D.M., Cozijnsen, A.J., Wilson, L.M., Pedras, M.S., Howlett, B.J., 2004. The sirodesmin biosynthetic gene cluster of the plant pathogenic fungus Leptosphaeria maculans. Mol. Microbiol. 53, 1307-1318.

Hamilton, A.J., Holdom, M.D., Jeavons, L., 1996. Expression of the $\mathrm{Cu}, \mathrm{Zn}$ superoxide dismutase of Aspergillus fumigatus as determined by immunochemistry and immunoelectron microscopy. FEMS Immunol. Med. Microbiol. 14, 95-102.

Hartmann, T., Dümig, M., Jaber, B.M., Szewczyk, E., Olbermann, P., Morschhäuser, J., Krappmann, S., 2010. Validation of a self-excising marker in the human pathogen Aspergillus fumigatus by employing the beta-rec/six site-specific recombination system. Appl. Environ. Microbiol. 76 (18), 6313-6317.

Holdom, M.D., Hay, R.J., Hamilton, A.J., 1996. The $\mathrm{Cu}, \mathrm{Zn}$ superoxide dismutases of Aspergillus flavus, Aspergillus niger, Aspergillus nidulans, and Aspergillus terreus: 
purification and biochemical comparison with the Aspergillus fumigatus $\mathrm{Cu}, \mathrm{Zn}$ superoxide dismutase. Infect. Immun. 64, 3326-3332.

Horiguchi, H., Yurimoto, H., Kato, N., Sakai, Y., 2001. Antioxidant system within yeast peroxisome. Biochemical and physiological characterization of CbPmp20 in the methylotrophic yeast Candida boidinii. J. Biol. Chem. 276, $14279-14288$

Johnson, J.R., Bruce, W.F., Dutcher, J.D., 1943. Gliotoxin, the antibiotic principle of Gliocladium fimbriatum. I. Production, physical and biological properties. J. Am. Chem. Soc. 65, 2005-2009.

Khalaj, V., Azarian, B., Enayati, S., Vaziri, B., 2011. Annexin C4 in A. fumigatus: a proteomics approach to understand the function. J. Proteomics 74, 1950-1958.

Kupfahl, C., Heinekamp, T. Geginat, G., Ruppert, T. Hartl, A., Hof, H., Brakhage, A.A. 2006. Deletion of the glip gene of Aspergillus fumigatus results in loss of gliotoxin production but has no effect on virulence of the fungus in a low-dose mouse infection model. Mol. Microbiol. 62, 292-302.

Kwon-Chung, K.J., Sugui, J.A., 2009. What do we know about the role of gliotoxin in the pathobiology of Aspergillus fumigatus? Med. Mycol. 47 (Suppl 1), S97-103.

Lambou, K., Lamarre, C., Beau, R., Dufour, N., Latge, J.P., 2010. Functional analysis of the superoxide dismutase family in Aspergillus fumigatus. Mol. Microbiol. 75, 910-923.

Lee, J.C., Straffon, M.J., Jang, T.Y., Higgins, V.J., Grant, C.M., Dawes, I.W., 2001. The essential and ancillary role of glutathione in Saccharomyces cerevisiae analysed using a grande gsh1 disruptant strain. FEMS Yeast Res. 1 (1), 57-65.

Lessing, F., Kniemeyer, O., Wozniok, I., Loeffler, J., Kurzai, O., Haertl, A., Brakhage, A.A., 2007. The Aspergillus fumigatus transcriptional regulator AfYap1 represents the major regulator for defense against reactive oxygen intermediates but is dispensable for pathogenicity in an intranasal mouse infection model. Eukaryot. Cell. 6, 2290-2302.

Losada, L., Ajayi, O., Frisvad, J.C., Yu, J., Nierman, W.C., 2009. Effect of competition on the production and activity of secondary metabolites in Aspergillus species. Med. Mycol. 47 (Suppl 1), S88-96.

Mabey, J.E., Anderson, M.J., Giles, P.F., Miller, C.J., Attwood, T.K., Paton, N.W., Bornberg-Bauer, E., Robson, G.D., Oliver, S.G., Denning, D.W., 2004. CADRE: the Central Aspergillus Data REpository. Nucleic Acids Res. 32, 401-405.

Narasaiah, K.V., Sashidhar, R.B., Subramanyam, C., 2006. Biochemical analysis of oxidative stress in the production of aflatoxin and its precursor intermediates. Mycopathologia 162, 179-189.

Nielsen, M.L., Albertsen, L., Lettier, G., Nielsen, J.B., Mortensen, U.H., 2006. Efficient PCR-based gene targeting with a recyclable marker for Aspergillus nidulans. Fungal Genet. Biol. 43, 54-64.

Niide, O., Suzuki, Y., Yoshimaru, T., Inoue, T., Takayama, T., Ra, C., 2006. Fungal metabolite gliotoxin blocks mast cell activation by a calcium- and superoxidedependent mechanism: implications for immunosuppressive activities. Clin. Immunol. 118, 108-116.

Nishida, S., Yoshida, L.S., Shimoyama, T., Nunoi, H., Kobayashi, T., Tsunawaki, S., 2005. Fungal metabolite gliotoxin targets flavocytochrome b558 in the activation of the human neutrophil NADPH oxidase. Infect. Immun. 73, 235244.

O’Brian, G.R., Georgianna, D.R., Wilkinson, J.R., Yu, J., Abbas, H.K., Bhatnagar, D., Cleveland, T.E., Nierman, W., Payne, G.A., 2007. The effect of elevated temperature on gene transcription and aflatoxin biosynthesis. Mycologia 99, 232-239.
Oosthuizen, J.L., Gomez, P., Ruan, J., Hackett, T.L., Moore, M.M., Knight, D.A., Tebbutt, S.J., 2011. Dual organism transcriptomics of airway epithelial cells interacting with conidia of Aspergillus fumigatus. PLoS One 6, e20527.

Patron, N.J., Waller, R.F., Cozijnsen, A.J., Straney, D.C., Gardiner, D.M., Nierman, W.C., Howlett, B.J., 2007. Origin and distribution of epipolythiodioxopiperazine (ETP) gene clusters in filamentous ascomycetes. BMC Evol. Biol. 7, 174.

Qiao, J., Kontoyiannis, D.P., Calderone, R., Li, D., Ma, Y., Wan, Z., Li, R., Liu, W., 2008 Afyap1, encoding a bZip transcriptional factor of Aspergillus fumigatus, contributes to the oxidative stress response but is not essential to the virulence of this pathogen in mice immunosuppressed by cyclophosphamide and triamcinolone. Med. Mycol. 46 (8), 773-782.

Rahman, I. Kode, A., Biswas, S.K. 2006. Assay for quantitative determination of glutathione and glutathione disulfide levels using enzymatic recycling method. Nat. Protocols 1, 3159-3165.

Raimondi, S., Uccelletti, D., Amaretti, A., Leonardi, A., Palleschi, C., Rossi, M., 2010 Secretion of Kluyveromyces lactis $\mathrm{Cu} / \mathrm{Zn}$ SOD: strategies for enhanced production. Appl. Microbiol. Biotechnol. 86, 871-878.

Rohlfs, M., Albert, M., Keller, N.P., Kempken, F., 2007. Secondary chemicals protect mould from fungivory. Biol. Lett. 3, 523-525.

Scharf, D.H., Remme, N., Heinekamp, T., Hortschansky, P., Brakhage, A.A., Hertweck, C., 2010. Transannular disulfide formation in gliotoxin biosynthesis and its role in self-resistance of the human pathogen Aspergillus fumigatus. J. Am. Chem. Soc 132, 10136-10141.

Schrettl, M., Kim, H.S., Eisendle, M., Kragl, C., Nierman, W.C., Heinekamp, T., Werner, E.R., Jacobsen, I., Illmer, P., Yi, H., Brakhage, A.A., Haas, H., 2008. SreA-mediated iron regulation in Aspergillus fumigatus. Mol. Microbiol. 70, 27-43.

Schrettl, M., Carberry, S., Kavanagh, K., Haas, H., Jones, G.W., O’Brien, J., Stephens, J. Fenelon, O., Nolan, A., Doyle, S., 2010. Self-protection against gliotoxin - a component of the gliotoxin biosynthetic cluster, GliT, completely protects Aspergillus fumigatus against exogenous gliotoxin. PLoS Pathogens 6, 6 .

Steiger, M.G., Vitikainen, M., Uskonsen, P., Brunner, K., Adam, G., Pakula, T., Penttilä, M., Saloheimo, M., Mach, R.L., Mach-Aigner, A.R., 2011. Transformation system for Hypocrea jecorina (Trichoderma reesei) that favors homlogous integration and employs reusable bidirectionally selectable markers. Appl. Environ. Microbiol. 77 (1), 114-121.

Sugui, J.A., Kim, H.S., Zarember, K.A., Chang, Y.C., Gallin, J.I., Nierman, W.C., KwonChung, K.J., 2008. Genes differentially expressed in conidia and hyphae of Aspergillus fumigatus upon exposure to human neutrophils. PLoS One 3, e2655.

Sutton, P., Newcombe, N.R., Waring, P., Mullbacher, A., 1994. In vivo immunosuppressive activity of gliotoxin, a metabolite produced by human pathogenic fungi. Infect. Immun. 62, 1192-1198.

Thön, M., Al Abdallah, Q., Hortschansky, P., Scharf, D.H., Eisendle, M., Haas, H. Brakhage, A.A., 2010. The CCAAT-binding complex coordinates the oxidative stress response in eukaryotes. Nucl. Acids Res. 38, 1098-1113.

Vickers, I., Reeves, E.P., Kavanagh, K.A., Doyle, S., 2007. Isolation, activity and immunological characterisation of a secreted aspartic protease, CtsD, from Aspergillus fumigatus. Protein Expr. Purif. 53, 216-224.

Waring, P., Sjaarda, A., Lin, Q.H., 1995. Gliotoxin inactivates alcohol dehydrogenase by either covalent modification or free radical damage mediated by redox cycling. Biochem. Pharmacol. 49, 1195-1201.

Watanabe, A., Kamei, K., Sekine, T., Waku, M., Nishimura, K., Miyaji, M., Tatsumi, K. Kuriyama, T., 2004. Effect of aeration on gliotoxin production by Aspergillus fumigatus in its culture filtrate. Mycopathologia 157, 245-254. 